\title{
Medical Device Document Writing - Correct Knowledge Process Outsourcing Partner will Be the Next-Gen Key to Success
}

\author{
Ashish Indani* \\ Complementary and Alternative Medicine, India \\ Submission: February 04, 2017; Published: February 13, 2017 \\ "Corresponding author: Ashish Indani, Complementary and Alternative Medicine, India, Eamil: ashishindani@gmail.com
}

\section{Introduction}

The medical device regulation is constantly evolving along with the evolving biomedical technology. Recently added guidance on 3D printed devices and customized medical implants in US, amendments in the Clinical Evaluation Report guidelines, Medical Device Reporting guidelines in EU and China and introduction of medical device rules 2017 in India are three major examples of the regulation's efforts keeping up with the technology. As a cascade phenomenon, such regulatory changes and additions impose binary effects the medical device industry. The affirmative side is that they provide complete clarity on the approach of regulation and thereby the requirements for their product's approvals. The negative side includes delay in documentation defers submission and as a consequence,often reduces the shelf life of thetechnology and its revenue premium. Example of angioplasty devices can be considered to support the delay statement.

Angioplasty is among the fastest evolving biomedical operative technology. After the invention of angioplasty balloon (Gurtzing Ref............), introduction of stent, drug eluting stent, various specialty balloons, self-expanding stents, use of different alloys, new drugs and new polymers in the stent,stent surface modifications, various specialty stents such as bifurcation stent and peripheral stent, drug eluting balloon, and recent thin strut bio-absorbable stents (Meril Life India 2014) has been journey just over approximately 50 years. In addition to these there are innumerable changes and introductions in the supporting and accessory devices like PTCA guide-wires. This entire development had been exponential in the last two decades. However, in this period, the industry also witnessed that the first and second generation devicesare completely off the market within less than 1 decade. The revenue premium generated from sale of these devices across the healthcare segment has exponentially fallen, inversely to the development. This example has set two major facts about the medical device industry that the high end technology has some exit point, meaning that the technology has its shelf life (Figure 1) Second, the lab to cath-lab delay in the PCI device life cycle is directly linked with its shelf life and profits.

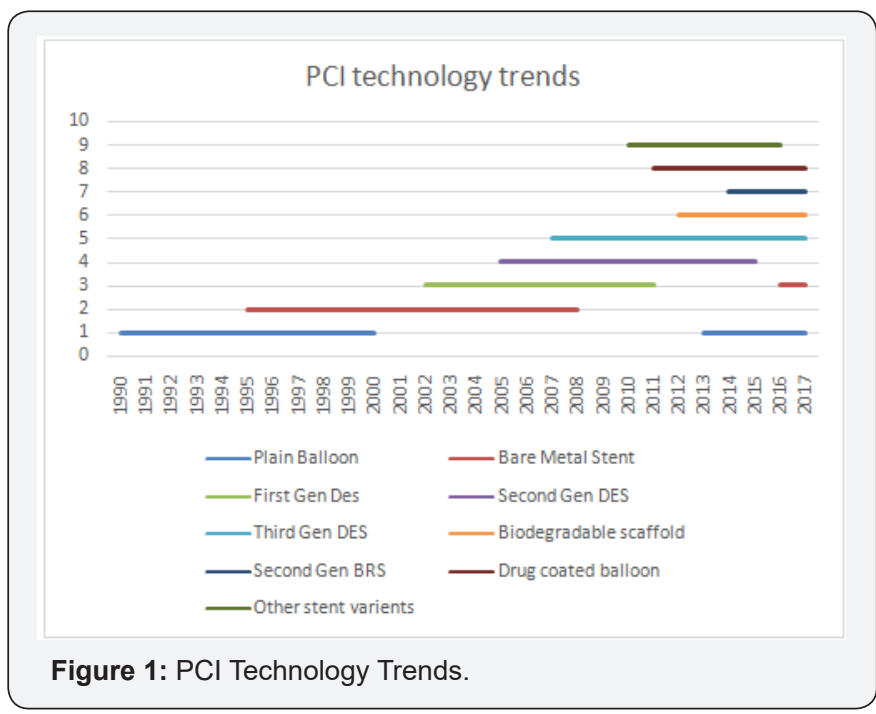

Even if the conclusion of PCI device case study appears to be specific, they are applicable to all high-end consumable devices. In the era of competition, increasing the technology shelf-life and end-user pricing are not in manufacturer's control. Therefore, the minimizing concept to market time and operational costs is the key. Among various contributors of such delay, one of the major contributor is regulatory process, mainly the time taken to compile and present a correct set of document. In addition, when a new regulation are implemented, various changes occur at ground level as in production floors, documents of operating procedures, quality management system etc. and supporting functions like training and logistics which add to the delay, resource management issues and the management stress. This article is an attempt to focus on the various effects of regulations and their changes on the medical device regulatory documentation and propose solution on some solutions to mitigate them. 


\section{Analysis of the Problem Components}

Geographic diversity of requirements of medico-technical documentation leads to the documentation diversity also. In addition, a wide range of technology variations, regulatory documentation writing is further modified by therapeutic areas and skills specific to type of document. In addition, the documents prepared need to be high quality and correct in first time, to avoid regulatory time lapses. All these the requirements are highly technical and demands specific skillsets. (Figure 2) Due to these all requirements, a multi-disciplinary team of experts is required for generation of most of the documents. A good team for regulatory and clinical regulatory documentation writing should be composed of biomedical engineers (biomedical-technical), Healthcare professionals including doctors (HCPs), graphic designers, IT professionals, graphic designers, statisticians, preclinical and animal study experts, Therapeutic area expert, medical device regulatory experts and literature search experts. In addition, support from marketing, finance and accounting, legal and some lay persons from the organization will be required time to time. The complexity of the team will be directly in line with the complexity of documents (Figure 3).

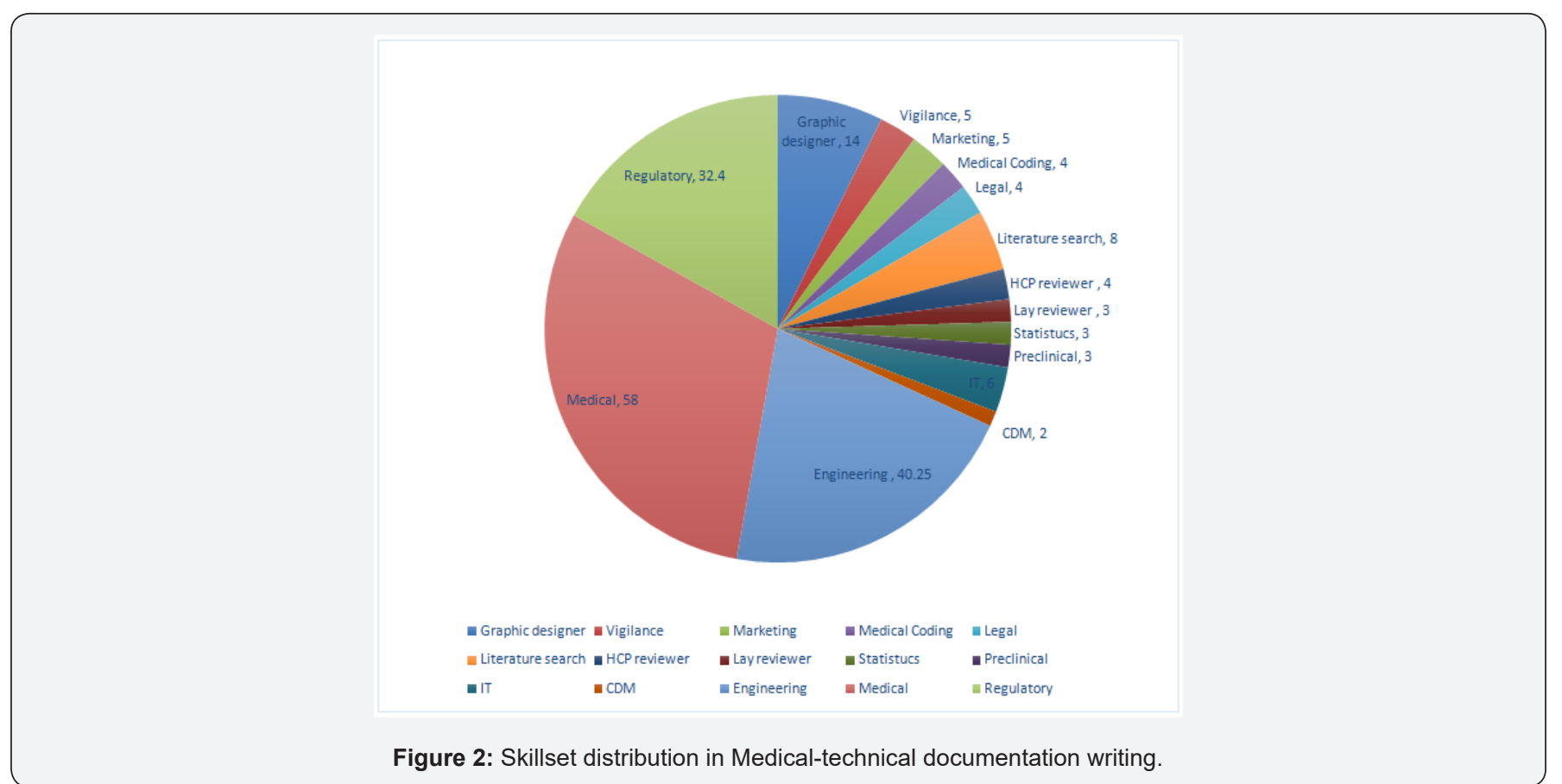

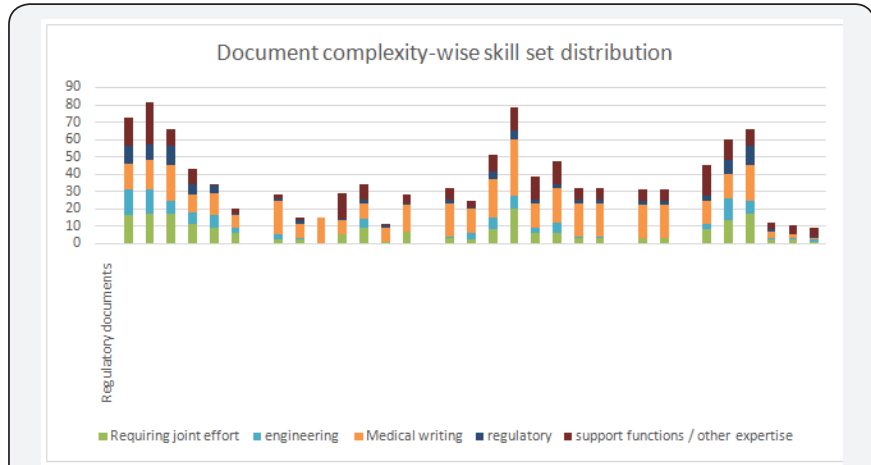

Figure 3: Document complexity-wise skillset analysis.

In addition to multidisciplinary team composition, within each discipline, there is micro-expertise. In the medical writing domain, there are more than 7 different micro-expertise based upon the skills namely plain language, labelling and IFU, medico-marketing, clinical regulatory, clinical and preclinical evidence, educational and publication. The diversity of regulatory requirements, language proficiency, and audience connect, and subsequently the skills can be estimated for each
documentation.Similar diversity can be observed regulatory expertise by domains, engineering expertise by technology used and Healthcare professionals by therapeutic areas. The demand of skills and unavailability of trained medical device professionals lead many organizations to adopt cross training the available resources in multiple skills and domains.

However, current trends demonstrate clustered distribution of efforts for documentation. (Figure 4) For example, for US FDA regulatory submission, submissions require labelling and IFU, patient IFU/ patient labeling, Substantial Equivalence Report, Post Marketing Surveillance Report including Patient Consequences Report, Clinical Research Regulatory documents and Publications. Apart from regulatory, medical writers are also expected to write marketing documents like brochure, campaigning and promotional material, educational medical communication for medical, paramedical and lay users as well as the sales force, most of which may be required in the submission dossier or before marketing authorization for staff training etc. As a new technology arises, the manufacturers will apply for marketing authorization in multiple geographies, 
which will multiply these efforts and hence demand for skilled professionals to deliver high quality documents in shot time.

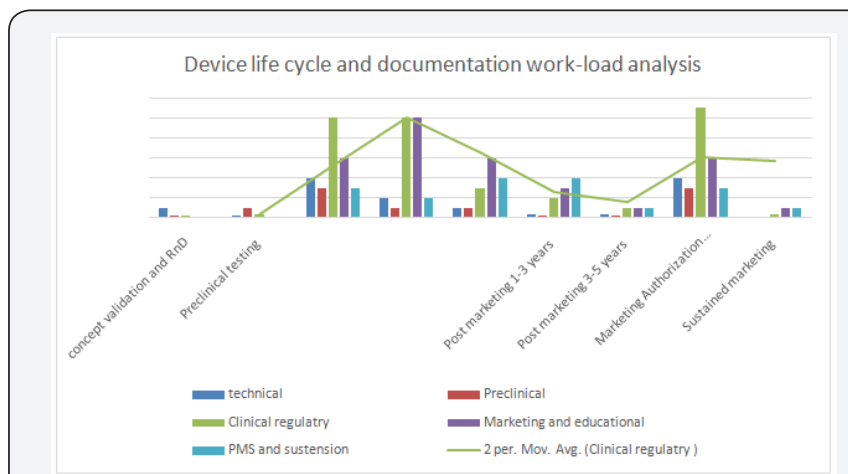

Figure 4: Device life cycle -wise documentation work-load analysis.

\section{Inference of the Problem Analysis}

From the discussion of the trends and business demands, the challenges are clear. First, the human resource management will be a challenge. In the current scenario, cross training of the staff is not a great solution. It will not only increase the stress and abhorrence in highly skilled professionals but also will lead to poor quality and operational delays in the peak period. Despite cross training or hiring the high skilled professionals, the second major challenge faced will be engagement of the associates. Due to high skills, niche and specific demands, the deployment index of micro-skill employees will be low and at the same time, the costs will be high. The combination of peak stress followed by underutilization of expertise will prove fatal and manufacturer's organization may face high attrition. The second inference is that the impact of regulatory changes will lead to delays in documentation and subsequently the submission. For further understanding, such delays are pertaining to the operational time, lapses due to resource unavailability of resources and lapses arising from quality issues. The operational time will further increase as the new regulation is implemented and that takes time for even micro-experts to understand and start incorporating in the existing processes.

\section{Solution and Approach Change}

The first major change in the approach is to reduce the lapses at each stage of product life cycle. This needs a transit from delivery orientation to result orientation. The approach of quality needs to change from multiple reviews to right at first time. Hence, the unit of operation needs to change from a trained person to an equipped team. This transformation will be brought in by use of automation and technology in generation of the document and the role of persons will be mostly to add value to the robotic work. This transformation will also gradually change an attitude of single person cross-trained in multiple functions to multiple experts contributing to a deliverable. This will generate multi-expert, multifunctional organogram, having micro-experts of all requisite disciplines. There will be a clubbed functioning, therefore. The clubbed functioning will allow the organizations to allow the experts to work on their own domain expertise and utilize their full potential.

However, the transformation of organizational structure may not be possible or feasible for all organizations and all the functions. Therefore, the second level of transformation will be in the approach to outsourcing. Classically, outsourcing was looked as a solution to reduce operational costs, certain types of liability and efforts on human resource management. However, the current trend of outsourcing have been adoptive. Beyond the classical reasoning, an adaptive reasoning of working with skilled people, matured processes and state-of-the-art technology has gained higher importance. There is now an evolving reasoning towards outsourcing of the regulatory documentation. Now, the outsourcing organization is being looked as an effective channel partnerfor development of knowledge, business and resource. (Figure 5) In this regards, a few organizations have already started filing joint patents, establishing joint SOPs and having profit sharing models.

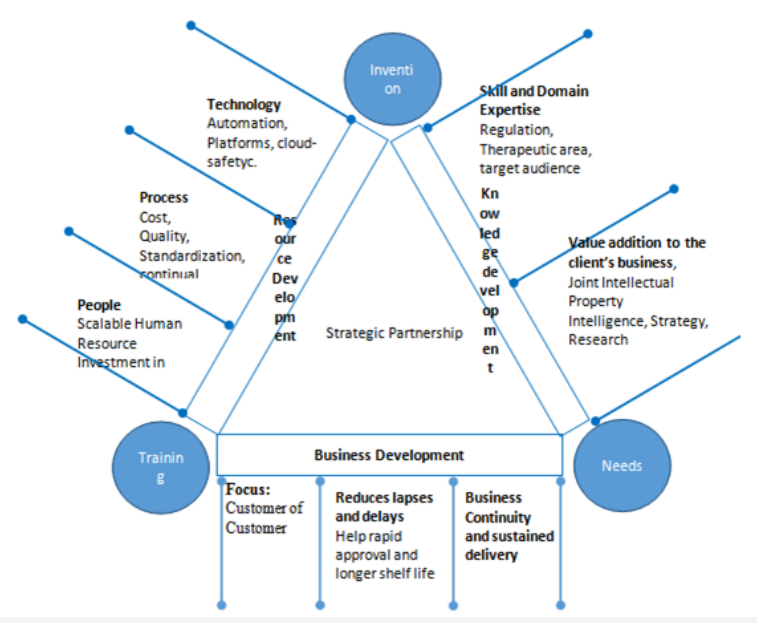

Figure 5: New approach to outsourcing qualifications. 
The transformation of approach to outsourcing from "vendor" to "partner" necessitates thorough evaluation of qualifications on transformed scale to find a correct outsourcing partner. The three major points

A. Talent pool Vs. resource scalability

B. Experience Vs. Domain expertise

C. Anticipatory business continuity strategy Vs Continuous delivery model

In major outsourcing destinations like India, South Asia and Latin America, talent pool is not a challenge. Skilled and trained individuals in technical, arts and medical domains are available at effective costs. However, an organization with a disposition to transform from "vendor" to partner will increase the scalability of the available resource by investing in training, innovation, exposure to the technology and encouraging learning and selfdevelopment. While these organizations invest in training and technological innovation, they maintain high production index to keep its associates adequately engaged and the operational costs low. This make the talent a scalable resource and the vendor a trustworthy partner.

Based upon the classical reasoning for outsourcing, the experience of the vendor in the required service area is considered a key parameter. However, in look for a correct channel partner, this definition needs to change from experience (quantity) to domain expertise (quantified quality). Instead of a vendor with experience of delivering 100 documents of a medical device, preferred partner will have 20 persons with experience of 10 documents each, having successfully accepted by various regulatory authorities.
Yet another point from classical qualifications of a vendor is time taken to disaster recovery. Most of the organizations in outsourcing domain propose an anticipatory planning for business continuity in case of a break-down due to a calamity. However, often these models are not validated and tested. An organization in transit from vendor to partner definitions in medical documentation has to maintain a continuous delivery model. This aspect of medical documentation outsourcing may not affect a business outcome, however, this will be a strategic parameter in view of accommodation of unanticipated regulatory requirements to be delivered in a short time.

\section{Conclusion and Opinions}

Evolving medical device regulations demands high skillset and multiple expertise for medical regulatory documentation preparation for marketing approval, marketing and sustenance. Evolution in technology is adding to the regulation continuously. For a manufacturing organization, who is usually busy with adoption of the changes on ground (in production facility and logistic etc.), for documentation becomes a major pain-point. Strategies such as cross training are not enough to balance availability and engagement of the human resource. Therefore, documentation process is better outsourced. However, with widespread transformation, the outsourcing approach needs to change from "vendor" to "partner" by both the counterparts of the outsourcing process. The outsourcing partner is a partner in knowledge, business and resource. For this change, a major qualification parameter definition change is required from Talent pool Vs. resource scalability, Experience Vs. Domain expertise, Anticipatory business continuity strategy Vs. Continuous delivery model. 